\title{
On the global evolution of vortex filaments, blobs, and small loops in $3 \mathrm{D}$ ideal flows
}

\author{
Luigi C. Berselli and Massimiliano Gubinelli \\ Dipartimento di Matematica Applicata "U.Dini", \\ Università di Pisa, Pisa I-56126 - ITALY \\ email: berselli@dma.unipi.it,m.gubinelli@dma.unipi.it \\ Tel: +39050 2219450, Fax: +390502219479
}

\begin{abstract}
We consider a wide class of approximate models of evolution of singular distributions of vorticity in three dimensional incompressible fluids and we show that they have global smooth solutions. The proof exploits the existence of suitable Hamiltonian functions.

The approximate models we analyze (essentially discrete and continuous vortex filaments and vortex loops) are related to some problem of classical physics concerning turbulence and also to the numerical approximation of flows with very high Reynolds number. Finally, we interpret our results as a basis to theoretical validation of numerical methods used in state-of-the-art computations of turbulent flows.
\end{abstract}

2000 MSC: 76B47, 76B03, 45K05.

Keywords. 3D Euler equations; vortex filaments, loops, and blobs.

\section{Introduction}

In this paper we consider the Euler equations describing the motion of an ideal (inviscid), incompressible and homogeneous fluid in $\mathbb{R}^{n}(n=2,3)$ :

$$
\frac{\partial \overrightarrow{\mathbf{u}}}{\partial t}+(\overrightarrow{\mathbf{u}} \cdot \nabla) \overrightarrow{\mathbf{u}}+\nabla p=\mathbf{0}
$$

and

$$
\nabla \cdot \overrightarrow{\mathbf{u}}=0,
$$

with the associated initial condition $\left.\overrightarrow{\mathbf{u}}\right|_{t=0}=\overrightarrow{\mathbf{u}}_{0}(\overrightarrow{\mathbf{x}})$, where $\overrightarrow{\mathbf{u}}=\left(\mathbf{u}^{1}, \ldots, \mathbf{u}^{n}\right)$ is the velocity field, while the scalar $p$ is the kinematic pressure.

To solve (1.1) and (1.2), the introduction of vorticity field is crucial. This quantity, that measures the "amount of rotation" inside the fluid, is responsible for the big differences between the $2 \mathrm{D}$ and the $3 \mathrm{D}$ case, see Wolibner [36] and Yudovich [38. If $n=2$, taking the curl of equation (1.1), one gets

$$
\frac{\partial \overrightarrow{\boldsymbol{\omega}}}{\partial t}+(\overrightarrow{\mathbf{u}} \cdot \nabla) \overrightarrow{\boldsymbol{\omega}}=\mathbf{0}
$$

where $\overrightarrow{\boldsymbol{\omega}}:=$ curl $\overrightarrow{\mathbf{u}}=\left(0,0, \partial_{1} \mathbf{u}^{2}-\partial_{2} \mathbf{u}^{1}\right)$, that is a pure transport equation. Then, a bounded initial vorticity remains bounded for all the positive times. Let us denote by $\overrightarrow{\mathbf{X}}_{t}(\overrightarrow{\mathbf{x}})$ the 
position at time $t$ of the fluid particle that at time 0 was in $\overrightarrow{\mathbf{x}}$. We have the following equation (equations for the characteristics)

$$
\frac{d \overrightarrow{\mathbf{X}}_{t}(\overrightarrow{\mathbf{x}})}{d t}=\overrightarrow{\mathbf{u}}\left(\overrightarrow{\mathbf{X}}_{t}(\overrightarrow{\mathbf{x}}), t\right)
$$

The vorticity field is simply transported along the trajectories of particles $\overrightarrow{\mathbf{X}}_{t}, t \geq 0$, remaining always perpendicular to the plane of motion. Beside, the vector $\overrightarrow{\boldsymbol{\omega}}$ having two component identically zero behaves essentially as a scalar.

When $n=3$, the situation becomes much more complicated. The vorticity associated to $\overrightarrow{\mathbf{u}}$ is a $3 \mathrm{D}$ vector field satisfying the following equation

$$
\frac{\partial \overrightarrow{\boldsymbol{\omega}}}{\partial t}+(\overrightarrow{\mathbf{u}} \cdot \nabla) \overrightarrow{\boldsymbol{\omega}}=(\overrightarrow{\boldsymbol{\omega}} \cdot \nabla) \overrightarrow{\mathbf{u}}
$$

In contrast to the $2 \mathrm{D}$ case, the vorticity $\overrightarrow{\boldsymbol{\omega}}$ is no longer conserved and the fundamental complication arising in three dimensions is the presence on the right hand side of $(\overrightarrow{\boldsymbol{\omega}} \cdot \nabla) \overrightarrow{\mathbf{u}}$, the "vortex stretching term," which is -roughly speaking- of order of $|\overrightarrow{\boldsymbol{\omega}}|^{2}$.

Some numerical simulations suggest that solutions of the ideal fluid equations may develop singularities and that the possible singularities may be detected by the blow-up of the vorticity field $\overrightarrow{\boldsymbol{\omega}}$, see Beale, Kato, and Majda [2] and references therein. Moreover, accurate direct numerical simulations of 3D turbulent fluids show that the regions where $|\overrightarrow{\boldsymbol{\omega}}(\overrightarrow{\mathbf{x}})|$ is big have an elongated "filament" shape, see for instance Bell and Markus [3], Vincent and Meneguzzi [35], and the reviews in Chorin [8] and Frisch [17. Thus, a consistent part of the current research activity is devoted to find suitable mathematical descriptions of these (and other geometric) vortex structures, see for instance Constantin 9]. In recent years also the use of the Lagrangian approach seems promising, to detect different intrinsic geometric properties of fluid flows, see Constantin [10.

We briefly recall some recent contributions on the study of these filaments, motivated also by application to the numerical simulation: Chorin [8] proposed a model based on selfavoiding random walks, using ideas coming from the theory of polymers; Gallavotti 18, suggested the introduction of non-smooth curves (like Brownian motions), to avoid some of the divergences arising in the equations; Lions [25] and Lions and Majda [26] proved a mean field result based on an approximate model of "nearly parallel" vortex filaments; Flandoli [15] introduced a probabilistic description and overcame certain divergences in the energy by considering filaments with a fractal cross-section. Furthermore, by using this model, authors in [16] have defined certain Gibbs measures, while Bessaih and Flandoli [5] proved a mean field result. Recent overview on vortex computational method can be found in Cottet and Koumoutsakos [14].

On the other hand, relevant advances in applied mathematics have been obtained in the study of $2 \mathrm{D}$ models for fluids (like the quasi-geostrophic equations) that replicate the 3D vorticity behavior via "analytic and geometric analogies," see Constantin, Majda, and Tabak 11] and Córdoba and Córdoba [12. This is obtained with a smoothing or a modification (with appropriate scaling properties) of the Biot-Savart law. Finally, very recently Cordoba et al. (see [13] and references therein) studied the breakdown of smooth tubes of vorticity and other singularities concentrated on small but elongated sets.

The basic goal of this paper is to show global existence of smooth solutions for models of line vortices, supplementing previous work in [4. In particular, we shall exhibit a Hamiltonian 
function. The main results will be derived by showing that the energy is conserved and also that the velocity induced by a singular distribution of vorticity is smooth enough. We shall consider rectifiable curves, but similar results can be also be proved for Hölder curves and some rough paths.

We shall also consider the vortex loop approximation (see Buttke [7] and Chorin [8]) for the equivalent equations of motion involving the impulse density. Also for this model we shall prove similar results of existence for large times of a smooth solutions.

Finally, we discuss the evolution for approximate equations in which a smooth vortex filament is approximated by a discretization and we shall connect the existence of solutions with an appropriate choice or refinement of interior points. This latter model is motivated being the simpler numerical approximation to the line vortex evolution problem.

Plan of the paper: The paper is organized as follows: In Section 2 we recall the motivation for the introduction of models with vorticity concentrated on singular sets together with classical methods to obtain integrable equations. Section 3 introduces a proper Hamiltonian and gives the main analytical results: conservation of energy and estimates of the velocity in terms of the energy that prove global existence of strong solutions. Section 4 uses the same technique to deal also with the problem of vortex loops in magnetization variables. Finally, in Section [5] a question connected with the resolution needed to simulate the behavior of a line vortex is put in the framework of the previous results.

\section{Vortex motion and approximate models}

To introduce the problem we shortly recall the discussion in Section 2 of [4, where the reader can find full details. Curves that are always parallel to the vorticity vector are known as vortex lines, and vortex lines passing through the points of a closed curve define a volume called vortex tube.

A vortex filament is a vortex tube that is immediately surrounded by irrotational fluid, even if the usage of this expression is neither uniform nor consistent. It can be also used to design infinitesimal vortex tubes or also patterns of vorticity that are not properly formed by vortex lines.

A line vortex is a singular distribution in which infinite vorticity is concentrated on a line, such that the circulation around a closed circuit threaded on by the line is finite. A line vortex is the result of a limiting process in which a vortex filament (of finite strength) is contracted to a curve, the strength $\Gamma>0$ being kept constant.

The laws of vortex motion, first formulated by Helmholtz [20, state that in an ideal fluid vortex lines and tubes move with the fluid and that the strength of each vortex tube (flux through any cross-section) remains constant in time.

Hence, we consider an ideal fluid with the vorticity $\overrightarrow{\boldsymbol{\omega}}$ concentrated on a smooth curve $\overrightarrow{\boldsymbol{\gamma}}$. Neglecting the transverse size of the filament, we can write the vorticity field $\overrightarrow{\boldsymbol{\omega}}$ generated

by $\vec{\gamma}$ (that is described parametrically as a continuous function $\vec{\gamma}:[0,1] \rightarrow \mathbb{R}^{3}$ such that $\vec{\gamma}(0)=\vec{\gamma}(1))$ and, formally,

$$
\left.\overrightarrow{\boldsymbol{\omega}}(\overrightarrow{\mathbf{x}}, t)=\Gamma \int_{0}^{1} \delta(\overrightarrow{\mathbf{x}}-\vec{\gamma}(\xi, t)) \vec{\gamma}_{\xi}(\xi, t) d \xi \quad \forall \overrightarrow{\mathbf{x}} \in \mathbb{R}^{3}, \forall t \in\right] 0, T[,
$$

where $\delta($.$) is the Dirac's delta function, \xi$ is the arc-length, the parameter $t$ represents the time, and $\vec{\gamma}_{\xi}$ is the derivative with respect to the arc-length. 
A discussion on the precise meaning of the formal vorticity field (2.1) and its distributional rigorous definition, can be found, for example, in the appendix of [4].

Remark 1 The above formula can also be written formally as the distribution

$$
\overrightarrow{\boldsymbol{\omega}}(\overrightarrow{\mathbf{x}}, t)=\Gamma \int_{0}^{1} \delta\left(\overrightarrow{\mathbf{x}}-\vec{\gamma}_{\xi}\right) d_{\xi} \vec{\gamma}
$$

meaning that the integral could be the Riemann-Stieltjes integral or also that included in a more general setting. In the sequel we shall not write explicitly the dependence of $\overrightarrow{\boldsymbol{\omega}}$ and $\overrightarrow{\mathbf{u}}$ on $\vec{\gamma}$ since we shall always consider vorticity (and corresponding velocity) "generated" by a distribution concentrated on a curve $\vec{\gamma}$.

It is well-known that the kinetic energy associated to (2.1) (in the sequel we shall denote by $\langle\overrightarrow{\mathbf{a}}, \overrightarrow{\mathbf{b}}\rangle$ the scalar product in $\mathbb{R}^{3}$ of the vectors $\overrightarrow{\mathbf{a}}$ and $\overrightarrow{\mathbf{b}}$ )

$$
E(t)=\frac{\Gamma^{2}}{8 \pi} \int_{0}^{1} \int_{0}^{1} \frac{1}{|\vec{\gamma}(\xi, t)-\vec{\gamma}(\eta, t)|}\left\langle\vec{\gamma}_{\xi}(\xi, t), \vec{\gamma}_{\eta}(\eta, t)\right\rangle d \xi d \eta
$$

is infinite for any reasonably smooth curve $\vec{\gamma}$. This explain the reason for the study of approximate models (as the Localized Induction Approximation (LIA) or the Rosenhead approximation, see Saffman [33) or for the same problem with curves supported by non-smooth lines (see Gallavotti [18] and recent results in [6]). To derive the evolution equation satisfied by a line vortex the essential tool is the Biot-Savart formula that gives a representation formula for the velocity, in terms of the vorticity:

$$
\overrightarrow{\mathbf{u}}(\overrightarrow{\mathbf{x}})=-\frac{1}{4 \pi} \int_{\mathbb{R}^{3}} \frac{(\overrightarrow{\mathbf{x}}-\overrightarrow{\mathbf{y}})}{|\overrightarrow{\mathbf{x}}-\overrightarrow{\mathbf{y}}|^{3}} \wedge \overrightarrow{\boldsymbol{\omega}}(\overrightarrow{\mathbf{y}}) d \overrightarrow{\mathbf{y}}
$$

where $\overrightarrow{\mathbf{a}} \wedge \overrightarrow{\mathbf{b}} \in \mathbb{R}^{3}$ is the vector (exterior) product of the vectors $\overrightarrow{\mathbf{a}}, \overrightarrow{\mathbf{b}} \in \mathbb{R}^{3}$. Inserting the expression (2.1) of the vorticity field in the Biot-Savart formula, using the equation for the characteristics (1.3), and imposing that the curve $\vec{\gamma}$ is transported by the velocity field $\overrightarrow{\mathbf{u}}$, we get the following equation

$$
\frac{\partial \vec{\gamma}}{\partial t}(\xi, t)=-\frac{\Gamma}{4 \pi} \int_{0}^{1} \frac{\vec{\gamma}(\xi, t)-\vec{\gamma}(\eta, t)}{|\vec{\gamma}(\xi, t)-\vec{\gamma}(\eta, t)|^{3}} \wedge \vec{\gamma}_{\eta}(\eta, t) d \eta
$$

This equation (relation between its solutions and weak solutions to the 3D Euler equations can be found -for instance- in the appendix of [4]) has been used for the first time by J.J. Thomson, who proposed a cut-off in the integral in order to control an infinite energy, see [33]. An approximation based on the Taylor expansion of the kernel in the singular integral gives the Localized Induction Approximation (LIA) solved by Hasimoto [19], while recent results in this direction are those by Klein and Majda 23, 24.

The de-singularized model studied analytically in [4] is that proposed by Rosenhead 32,

$$
\frac{\partial \vec{\gamma}}{\partial t}=-\frac{\Gamma}{4 \pi} \int_{0}^{1} \frac{\vec{\gamma}(\xi, t)-\vec{\gamma}(\eta, t)}{\left[(\vec{\gamma}(\xi, t)-\vec{\gamma}(\eta, t))^{2}+\mu^{2}\right]^{3 / 2}} \wedge \vec{\gamma}_{\eta}(\eta, t) d \eta,
$$

for some $\mu>0$. The choice was motivated by the fact that (2.2) gives advantages for numerical integration and, starting by Moore [30, it has been used in the applied context for some calculation related to aircraft trailing vortices. 


\subsection{On the Rosenhead model}

Regarding the Rosenhead model, a first analytical result has been recently proved in [4. In that reference is shown (as a particular case) that if the initial curve is smooth and closed then the initial value problem is well-posed in natural Sobolev spaces. In the sequel we shall use the customary $W^{k, p}$ and $H^{k}=W^{k, 2}$ spaces, see Adams [1]. To our knowledge the following is essentially the first rigorous existence and uniqueness result, known for this model.

Theorem 1 [See [4]] Let $\vec{\gamma}_{0}(\xi) \in H_{\#}^{1}(0,1)$, where $H_{\#}^{1}(0,1)$ denotes the subset of closed curves $\mathbb{R} \mapsto \mathbb{R}^{3}$ belonging to the Sobolev space $H^{1}(0,1)$. Then, there exist a strictly positive $T^{*} \leq T$ and a unique curve $\vec{\gamma}$ such that

$$
\begin{aligned}
& \text { a) } \vec{\gamma} \in W^{1, \infty}\left(0, T ; L^{2}(0,1)\right) \cap L^{\infty}\left(0, T ; H_{\#}^{1}(0,1)\right) \\
& \text { b) } \vec{\gamma}(\xi, t)=\vec{\gamma}_{0}(\xi)-\frac{\Gamma}{4 \pi} \int_{0}^{t} \int_{0}^{1} \frac{\vec{\gamma}(\xi, \tau)-\vec{\gamma}(\eta, \tau)}{\left[(\vec{\gamma}(\xi, \tau)-\vec{\gamma}(\eta, \tau))^{2}+\mu^{2}\right]^{3 / 2}} \wedge \vec{\gamma}_{\eta}(\tau) d \eta d \tau,
\end{aligned}
$$

that is a strong solution to (2.2) in the time interval $\left[0, T^{*}\right)$.

In addition the time $T^{*}>0$ may be estimated from below and it depends just on the $H^{1}$-norm of the initial datum.

A similar result has been recently proved in a more general stochastic context in [6] with a different target and weaker hypotheses on the smoothness of the curve $\vec{\gamma}$. Anyway, in both papers the question of the global solvability left open and some continuation criteria involving the length of the curve itself were derived.

Our main goal is now to show that, for a class of regularizations (including the one above) the above local theorem is in fact global.

\subsection{A class of regularized problems}

An interesting class of regularized evolution equations can be defined as follows. Let $\varphi: \mathbb{R}^{3} \rightarrow$ $\mathbb{R}$ be a scalar function and define the velocity field $\overrightarrow{\mathbf{u}}$ associated to the vortex filament $\vec{\gamma}$ as

$$
\overrightarrow{\mathbf{u}}(\overrightarrow{\mathbf{x}}):=(\operatorname{curl} \varphi) * \overrightarrow{\boldsymbol{\omega}}=\int_{0}^{1} \nabla \varphi(\overrightarrow{\mathbf{x}}-\vec{\gamma}(\xi)) \wedge \vec{\gamma}_{\xi}(\xi) d \xi
$$

where the convolution $*$ has to be intended in the sense of distributions. Formally, this formula is such that curl $\overrightarrow{\mathbf{u}}=\overrightarrow{\boldsymbol{\omega}}$.

On the kernel $\varphi$ we impose the following conditions that will allow the energy to be well-defined.

Hypothesis A. In the sequel we shall assume that:

- The function $\varphi$ is even:

$$
\varphi(-\overrightarrow{\mathbf{x}})=\varphi(\overrightarrow{\mathbf{x}})
$$

- The function $\varphi$ has a real and non-negative Fourier transform:

$$
\widehat{\varphi}(\overrightarrow{\mathbf{k}}):=\int_{\mathbb{R}^{3}} \mathrm{e}^{i\langle\overrightarrow{\mathbf{k}}, \overrightarrow{\mathbf{x}}\rangle} \varphi(\overrightarrow{\mathbf{x}}) d \overrightarrow{\mathbf{x}} \geq 0 \quad \forall \overrightarrow{\mathbf{k}} \in \mathbb{R}^{3}
$$


- The Fourier transform $\widehat{\varphi}(\overrightarrow{\mathbf{k}})$ is integrable over $\mathbb{R}^{3}$ :

$$
\int_{\mathbb{R}^{3}} \widehat{\varphi}(\overrightarrow{\mathbf{k}}) d \overrightarrow{\mathbf{k}}<+\infty
$$

- The function $\varphi$ is smooth enough, in order that

$$
\int_{\mathbb{R}^{3}}\left(1+|\overrightarrow{\mathbf{k}}|^{2}\right)^{2} \widehat{\varphi}(\overrightarrow{\mathbf{k}}) d \overrightarrow{\mathbf{k}}<\infty
$$

These assumptions are satisfied by a wide range of function $\varphi$, covering also well-known cases with a physical meaningful interpretation.

Remark 2 (An explicit relevant example) The function

$$
\varphi_{R}(\overrightarrow{\mathbf{x}})=\frac{\Gamma}{\left(|\overrightarrow{\mathbf{x}}|^{2}+\mu^{2}\right)^{1 / 2}}
$$

for some $\mu>0$ would be a natural candidate satisfying all the above assumptions. Assumption (A.1) is trivially satisfied. Regarding the assumption (A.3), the Fourier Transform may be expressed in terms of a Bessel function of the second kind $y(z)=\operatorname{Bessel}(1, z)$, defined as solution of the differential equation $z^{2} y^{\prime \prime}+z y^{\prime}-\left(z^{2}+1\right) y=0$. The condition on the nonnegativity of the Fourier transform can be verified by a direct computation:

$$
\begin{aligned}
\widehat{\varphi}_{R}(\overrightarrow{\mathbf{k}}) & =\Gamma \int_{\mathbb{R}^{3}} \frac{\mathrm{e}^{i\langle\overrightarrow{\mathbf{k}}, \overrightarrow{\mathbf{x}}\rangle}}{\left(|\overrightarrow{\mathbf{x}}|^{2}+\mu^{2}\right)^{1 / 2}} d \overrightarrow{\mathbf{x}} \\
& =\Gamma \pi^{-1 / 2} \int_{0}^{\infty} d t \int_{\mathbb{R}^{3}} \frac{\mathrm{e}^{i\langle\mathbf{k}, \overrightarrow{\mathbf{x}}\rangle-t|\overrightarrow{\mathbf{x}}|^{2}-t \mu^{2}}}{t^{1 / 2}} d \overrightarrow{\mathbf{x}} \\
& =\Gamma \pi \int_{0}^{\infty} \frac{\mathrm{e}^{-|\overrightarrow{\mathbf{k}}|^{2} /(4 t)-t \mu^{2}}}{t^{2}} d t \geq 0
\end{aligned}
$$

where we used the fact that

$$
\lambda^{-1 / 2}=\pi^{-1 / 2} \int_{0}^{\infty} e^{-\lambda t} t^{-1 / 2} d t
$$

and employed the explicit expression of the Fourier transform of the Gaussian kernel.

Regarding (A.3) the integral over $\mathbb{R}^{3}$ can be split into the inner and outer part. The first one is bounded since the function $\widehat{\varphi}_{R}(\overrightarrow{\mathbf{k}})$ is $O\left(|\mathbf{k}|^{-2}\right)$ near the origin, hence the inner integral converges. On the other hand, the decay at infinity necessary to show the convergence of the outer integral comes directly by observing that $\widehat{\varphi}_{R}(\overrightarrow{\mathbf{k}})=2 \mu^{2} \operatorname{Bessel}(1,2|\overrightarrow{\mathbf{k}}| \mu)(\mu|\overrightarrow{\mathbf{k}}|)^{-1}$. The fast decay at infinity (enough to have both (A.3) and (A.4) satisfied) derives from the properties of this special-function.

In this case we obtain, by using as kernel the function $\varphi_{R}$, exactly the Rosenhead model where the velocity field is given by the equation:

$$
\overrightarrow{\mathbf{u}}(\overrightarrow{\mathbf{x}})=\Gamma \int_{0}^{1} \frac{\overrightarrow{\mathbf{x}}-\vec{\gamma}(\xi)}{\left(|\overrightarrow{\mathbf{x}}-\vec{\gamma}(\xi)|^{2}+\mu^{2}\right)^{3 / 2}} \wedge \vec{\gamma}_{\xi}(\xi) d \xi
$$


The evolution problem can be set up in different Banach spaces of closed paths where local solution exists and are unique. For example we can have solution for initial condition in the Sobolev space $H_{\#}^{1}(0,1)$, in the space of Hölder continuous with exponent greater than $1 / 2$, and also in some spaces of rough-paths, see 4, 6.

Details of the proofs (and some specific assumptions) depend however on the functional setting, so we will show the proofs in the case of solutions living in $H_{\#}^{1}(0,1)$, using the framework of Theorem 1 .

\section{$3 \quad$ Energy and global solutions}

For the evolution problem

$$
\frac{\partial}{\partial t} \vec{\gamma}(\xi, t)=\overrightarrow{\mathbf{u}}(\vec{\gamma}(\xi, t), t)
$$

with velocity given by (2.3) we can identify a function which plays the röle of a "kinetic" energy.

Definition 1 (Kinetic energy.) The function below $\mathcal{H}: \vec{\gamma} \mapsto \mathbb{R}$, defined on the space of smooth curves, is the "kinetic energy" for the smoothed evolution problem associated to (3.1)

$$
\mathcal{H}(\vec{\gamma}):=\frac{1}{2} \int_{0}^{1} \int_{0}^{1} \varphi(\vec{\gamma}(\xi)-\vec{\gamma}(\eta))\left\langle\vec{\gamma}_{\xi}(\xi), \vec{\gamma}_{\eta}(\eta)\right\rangle d \xi d \eta
$$

The main result we shall prove is that $\mathcal{H}(\vec{\gamma})$ is constant in time. Then, the existence of the energy allows to exploit a-priori estimates to have global existence for initial data with finite energy.

Remark 3 This particular expression for the energy has been discussed also by Marsden and Weinstein [29] and Holm [21], they show that on the space of closed curves in $\mathbb{R}^{3}$ there exists a natural Poisson structure and that, formally, with respect to this structure the function (3.2) is the Hamiltonian function which generates the flow described by (3.1).

First, we show that the function $\mathcal{H}(\vec{\gamma})$ is well-defined.

Lemma 1 For each smooth enough curve $\vec{\gamma}$, it holds that $0 \leq \mathcal{H}(\vec{\gamma})<+\infty$.

Proof. In terms of Fourier variables, the energy $\mathcal{H}(\vec{\gamma})$ can be written as

$$
\begin{aligned}
\mathcal{H}(\vec{\gamma}) & =\frac{1}{2(2 \pi)^{3}} \int_{0}^{1} \int_{0}^{1} \int_{\mathbb{R}^{3}} \widehat{\varphi}(\overrightarrow{\mathbf{k}}) \mathrm{e}^{-i\langle\overrightarrow{\mathbf{k}}, \vec{\gamma}(\xi)-\vec{\gamma}(\eta)\rangle} \vec{\gamma}_{\xi}(\xi) \vec{\gamma}_{\eta}(\eta) d \overrightarrow{\mathbf{k}} d \xi d \eta \\
& =\frac{1}{2(2 \pi)^{3}} \int_{\mathbb{R}^{3}} \widehat{\varphi}(\overrightarrow{\mathbf{k}})\left|\int_{0}^{1} \mathrm{e}^{i\langle\overrightarrow{\mathbf{k}}, \vec{\gamma}(\xi)\rangle} \vec{\gamma}_{\xi}(\xi) d \xi\right|^{2} d \overrightarrow{\mathbf{k}}
\end{aligned}
$$

which, thanks to the assumption on the non-negativity of the Fourier transform of $\varphi$, proves that the energy is a non-negative quantity. Moreover, for any $\vec{\gamma} \in H_{\#}^{1}(0,1)$ we have the obvious estimate

$$
\left|\int_{0}^{1} \mathrm{e}^{i\langle\overrightarrow{\mathbf{k}}, \vec{\gamma}(\xi)\rangle} \vec{\gamma}_{\xi}(\xi) d \xi\right| \leq \int_{0}^{1}\left|\vec{\gamma}_{\xi}(\xi)\right| d \xi,
$$


actually we observe that sharper estimates are possible, even if they are not needed at this stage. Finally, we have proved that

$$
\mathcal{H}(\vec{\gamma}) \leq \frac{1}{2(2 \pi)^{3}}\left(\int_{\mathbb{R}^{3}} \widehat{\varphi}(\overrightarrow{\mathbf{k}}) d \overrightarrow{\mathbf{k}}\right)\left(\int_{0}^{1}\left|\vec{\gamma}_{\xi}(\xi)\right| d \xi\right)^{2} .
$$

The final observation is that both integrals are finite (use assumption (A.3) and the fact that $\vec{\gamma}$ is rectifiable a curve), showing that $\mathcal{H}(\vec{\gamma})$ is a well-defined energy for any $\vec{\gamma} \in H_{\#}^{1}(0,1)$.

Next, we show that the function $\mathcal{H}(\vec{\gamma})$ behaves as a constant of motion if $\vec{\gamma}$ evolves under the flow associated to $\overrightarrow{\mathbf{u}}$.

Lemma 2 Let $\vec{\gamma}(t, \xi)$ be a local smooth (as those of Th. 11) solution of the problem (3.1), then

$$
\frac{d \mathcal{H}(\vec{\gamma}(t, \xi))}{d t}=0
$$

Proof. We shall show by an explicit computation that the energy $\mathcal{H}(\vec{\gamma})$ is invariant and the completely anti-symmetric Levi-Civita tensor $\epsilon_{i j k}$ will be used to write, with the Einstein repeated indices convention, that

$$
\mathbf{u}^{i}(\overrightarrow{\mathbf{x}})=\epsilon_{i j k} \int_{0}^{1} \nabla^{j} \varphi(\overrightarrow{\mathbf{x}}-\vec{\gamma}(\xi)) \gamma_{\xi}^{k}(\xi) d \xi .
$$

Then, with explicit vector notations, we have:

$$
\begin{aligned}
\frac{d \mathcal{H}(\vec{\gamma}(t, \xi))}{d t}=\int_{0}^{1} & \int_{0}^{1} \nabla^{k} \varphi(\vec{\gamma}(t, \xi)-\vec{\gamma}(t, \eta)) \partial_{t} \gamma^{k}(t, \xi) \gamma_{\xi}^{i}(t, \xi) \gamma_{\eta}^{i}(t, \eta) d \xi d \eta \\
& +\int_{0}^{1} \int_{0}^{1} \varphi(\vec{\gamma}(t, \xi)-\vec{\gamma}(t, \eta)) \partial_{t} \gamma_{\xi}^{i}(t, \xi) \gamma_{\eta}^{i}(t, \eta) d \xi d \eta
\end{aligned}
$$

where, for simplicity, $\partial_{t} \vec{\gamma}(t):=\partial \vec{\gamma}(t) / \partial t$.

By integrating by parts the $\xi$-integral in the second term we get

$$
\begin{aligned}
\frac{d \mathcal{H}(\vec{\gamma}(t, \xi))}{d t}= & \int_{0}^{1} \int_{0}^{1} \nabla^{k} \varphi(\vec{\gamma}(t, \xi)-\vec{\gamma}(t, \eta)) \partial_{t} \gamma^{k}(t, \xi) \gamma_{\xi}^{i}(t, \xi) \gamma_{\eta}^{i}(t, \eta) d \xi d \eta \\
& -\int_{0}^{1} \int_{0}^{1} \nabla^{k} \varphi(\vec{\gamma}(t, \xi)-\vec{\gamma}(t, \eta)) \partial_{t} \gamma^{i}(t, \xi) \gamma_{\xi}^{k}(t, \xi) \gamma_{\eta}^{i}(t, \eta) d \xi d \eta \\
= & \epsilon_{c a b} \epsilon_{c i j} \int_{0}^{1} \int_{0}^{1} \nabla^{a} \varphi(\vec{\gamma}(t, \xi)-\vec{\gamma}(t, \eta)) \partial_{t} \gamma^{i}(t, \xi) \gamma_{\xi}^{j}(t, \xi) \vec{\gamma}_{\eta}^{b}(t, \eta) d \xi d \eta
\end{aligned}
$$

where we used the fact that

$$
\epsilon_{c a b} \epsilon_{c i j}=\delta_{a i} \delta_{b j}-\delta_{a j} \delta_{b i} .
$$

Next, by definition of $\overrightarrow{\mathbf{u}}$ we have

$$
\begin{aligned}
\frac{d \mathcal{H}(\vec{\gamma}(t, \xi))}{d t} & =\epsilon_{c i j} \int_{0}^{1} \mathbf{u}^{c}(\vec{\gamma}(t, \xi)) \partial_{t} \boldsymbol{\gamma}^{i}(t, \xi) \boldsymbol{\gamma}_{\xi}^{j}(t, \xi) d \xi \\
& =\epsilon_{c i j} \int_{0}^{1} \mathbf{u}^{c}(\vec{\gamma}(t, \xi)) \mathbf{u}^{i}(\vec{\gamma}(t, \xi)) \boldsymbol{\gamma}_{\xi}^{j}(t, \xi) d \xi \\
& =0
\end{aligned}
$$


where we used in sequence the equation (3.1) of motion for $\vec{\gamma}(t)$ and the complete antisymmetry of the tensor $\epsilon_{i j k}$.

The next step is to show that if the kinetic energy is bounded, then the velocity $\overrightarrow{\mathbf{u}}$ associated to the evolution problem is smooth. In particular, it will follow that the velocity induced by vorticity concentrated over a $H^{1}$-curve is very regular. The regularity of the smoothing kernel is inherited by the velocity $\overrightarrow{\mathbf{u}}$ even if the framework is that of a singular problem.

Lemma 3 For any $0 \leq n \in \mathbb{N}$, we have the bound

$$
\left\|\nabla^{n} \overrightarrow{\mathbf{u}}\right\|_{L^{\infty}} \leq \frac{1}{2 \pi^{3 / 2}}\left[\int_{\mathbb{R}^{3}}|\overrightarrow{\mathbf{k}}|^{2(1+n)} \widehat{\varphi}(\overrightarrow{\mathbf{k}}) d \overrightarrow{\mathbf{k}}\right]^{1 / 2} \mathcal{H}^{1 / 2}(\vec{\gamma})
$$

provided that the integral $\int_{\mathbb{R}^{3}}|\overrightarrow{\mathbf{k}}|^{2(1+n)} \widehat{\varphi}(\overrightarrow{\mathbf{k}}) d \overrightarrow{\mathbf{k}}$ is finite.

Proof. The proof follows easily by using Cauchy-Schwartz inequality. In fact, it follows that

$$
\begin{aligned}
|\overrightarrow{\mathbf{u}}| & =\frac{1}{(2 \pi)^{3}}\left|\int_{\mathbb{R}^{3}} \mathrm{e}^{i\langle\overrightarrow{\mathbf{k}}, \overrightarrow{\mathbf{x}}\rangle} \widehat{\varphi}(\overrightarrow{\mathbf{k}})(i \overrightarrow{\mathbf{k}}) \wedge \int_{0}^{1} \mathrm{e}^{-i\langle\overrightarrow{\mathbf{k}}, \vec{\gamma}(\xi)\rangle} \vec{\gamma}(\xi) d \xi d \overrightarrow{\mathbf{k}}\right| \\
& \leq \frac{1}{(2 \pi)^{3}}\left[\int_{\mathbb{R}^{3}}|\overrightarrow{\mathbf{k}}|^{2} \widehat{\varphi}(\overrightarrow{\mathbf{k}}) d \overrightarrow{\mathbf{k}}\right]^{1 / 2}\left[\int_{\mathbb{R}^{3}} \widehat{\varphi}(\overrightarrow{\mathbf{k}})\left|\int_{0}^{1} \mathrm{e}^{-i\langle\overrightarrow{\mathbf{k}}, \vec{\gamma}(\xi)\rangle} \vec{\gamma}(\xi) d \xi\right|^{2} d \overrightarrow{\mathbf{k}}\right]^{1 / 2} \\
& =\frac{1}{2 \pi^{3 / 2}}\left[\int_{\mathbb{R}^{3}}|\overrightarrow{\mathbf{k}}|^{2} \widehat{\varphi}(\overrightarrow{\mathbf{k}}) d \overrightarrow{\mathbf{k}}\right]^{1 / 2} \mathcal{H}^{1 / 2}(\vec{\gamma}),
\end{aligned}
$$

and in a similar fashion we can bound all the derivatives of $\overrightarrow{\mathbf{u}}$, provided that $\varphi$ is smooth enough:

$$
\left\|\nabla^{n} \overrightarrow{\mathbf{u}}\right\|_{L^{\infty}} \leq \frac{1}{2 \pi^{3 / 2}}\left[\int_{\mathbb{R}^{3}}|\overrightarrow{\mathbf{k}}|^{2(1+n)} \widehat{\varphi}(\overrightarrow{\mathbf{k}}) d \overrightarrow{\mathbf{k}}\right]^{1 / 2} \mathcal{H}^{1 / 2}(\vec{\gamma})
$$

Essentially we shall use this lemma just for $n=1$ to prove global existence of solutions, hence assumption (A.4) will be enough. On the other hand, to prove higher regularity of $\overrightarrow{\mathbf{u}}$ these extra conditions on $\widehat{\varphi}$ are needed.

Theorem 2 The evolution problem (3.1) has a unique global solution for any initial condition in $H_{\#}^{1}(0,1)$.

Proof. Denote $\overrightarrow{\boldsymbol{\psi}} \in H_{\#}^{1}(0,1)$ the initial condition, then we already know that local solution $\vec{\gamma}(t, \xi)$ exists in a (possibly small) time interval $t \in\left[0, T^{*}\left[\right.\right.$, depending on the $L^{2}$-norm of $\overrightarrow{\boldsymbol{\psi}}_{\xi}$, recall Theorem 10 However, by taking the derivative of (3.1) with respect to $\xi$, and with and integration over $[0, t]$, we have now the additional a-priori estimate $(\|\cdot\|$ denotes the $L^{2}(0,1)$-norm)

$$
\begin{aligned}
\left\|\vec{\gamma}_{\xi}(t, \xi)\right\| & \leq\left\|\vec{\gamma}_{\xi}(0)\right\|+\int_{0}^{t}\|\nabla \overrightarrow{\mathbf{u}}(s)\|\left\|\vec{\gamma}_{\xi}(s, \xi)\right\| d s \\
& \leq\left\|\vec{\gamma}_{\xi}(0)\right\|+C \int_{0}^{t} \mathcal{H}^{1 / 2}(\vec{\gamma}(s))\left\|\vec{\gamma}_{\xi}(s, \xi)\right\| d s \\
& =\left\|\vec{\gamma}_{\xi}(0)\right\|+C \mathcal{H}^{1 / 2}(\vec{\gamma}(0)) \int_{0}^{t}\left\|\vec{\gamma}_{\xi}(s, \xi)\right\| d s
\end{aligned}
$$


where the fist line is an easy bound for the norm of the solution, the second line comes from Lemma 3] and the last line from the fact that the energy is constant along solutions (as stated in Lemma 2)

Then, by using the Gronwall inequality we have

$$
\sup _{t \in[0, T]}\left\|\vec{\gamma}_{\xi}(t, \xi)\right\| \leq\left\|\vec{\gamma}_{\xi}(0)\right\| \mathrm{e}^{C \mathcal{H}^{1 / 2}(\vec{\gamma}(0)) T}
$$

which guarantees existence of global solution, since any local solution, having the $H^{1}(0,1)$ norm uniformly bounded can be uniquely continued up to any positive time $T$.

\subsection{Hölder initial conditions}

In [6] the authors prove that for sufficiently regular functions $\varphi$ the vortex filament equation has local solutions for initial conditions $\vec{\gamma}(0)$ which are Hölder continuous functions of exponent $\alpha$ with $\alpha \geq 1 / 2$. In this case, the line integrals of the form

$$
\int_{0}^{1}\left\langle\overrightarrow{\mathbf{f}}(\vec{\gamma}(t, \xi)), \vec{\gamma}_{\xi}(t, \xi)\right\rangle d \xi=\int_{0}^{1}\left\langle\overrightarrow{\mathbf{f}}(\vec{\gamma}(t, \xi)), d_{\xi} \vec{\gamma}(t, \xi)\right\rangle
$$

must be understood as limits of Riemann sums: such limit exists if the function $\overrightarrow{\mathbf{f}}: \mathbb{R}^{3} \rightarrow \mathbb{R}^{3}$ is at least $C^{1}$ and this process defines the integral in the sense of Young 37.

Moreover, in the same paper, the authors extend the local existence result, to a class of initial conditions living in a space of rough paths (see the book of Lyons for more details 27. 28] ) that are special class of Hölder paths with some additional structure. For rough paths it is possible to define the line integrals (as those in eq. (3.4)) by means of natural "renormalized" Riemann sums. In this way it has been proved that the filament equation (with sufficient regularity of the kernel $\varphi$ ) admits local solutions starting from almost every 3D Brownian loop (i.e. curves chosen accordingly to the Wiener measure restricted to the subspace of closed curves).

The proof of the constancy of the energy extends also to these different functional setting and the existence of global solutions can be proved along the same strategy used for $H^{1}$ paths. We leave the technical details to the interested reader, since they are outside the scope of the present paper. For example, the extension of Lemma 2 to Hölder solutions can be done using convergence of approximating discretizations and the computations contained in Sec. 5.

Remark 4 The relevance of this additional result can be seen in the light of the K41 theory and the Onsager conjecture on possible singularities in the sense of $C^{0, \alpha}$ velocity fields. This may suggest that velocity behaves as Hölder continuous velocity field (see [17]), hence as a singularity diffused all over the fluid. More recent developments link intermittency with concentration of singularities on small sets, described by Hölder-hence (multi-)fractals- sets, see [17, 18].

\section{Impulse formulation}

In this section we consider another singular distribution of vorticity that is well-known and useful in the scientific computing, since it involves vector fields (as the "magnetization") that are possibly with non-vanishing divergence. In particular, we shall consider Buttke loops that 
(according to the terminology of Chorin [8]) are small loops of vorticity in an irrotational background, which evolve according to 3D Euler equation. The first study of the kinematic interaction of an immersed body in an inviscid irrotational flow dates back to Kelvin [22] and his analysis was based on the study of fluid impulse. It was in that paper that he introduced a model of "core-less vortices" in order to explain some experimental facts on eddy formation at the boundary. The interaction of a vortex ring (line) in an inviscid flow has been also studied by Roberts 31, by using as canonical variables the position of the centroid of each ring and its impulse (called by Roberts "momentum of vorticity"). A proper Hamiltonian formulation of the 3D Euler equations have been discovered independently by different authors, see for instance Osedelets 34 for a development in a continuum setting, with canonical variables the position and the impulse density. Then, around 1990 Buttke [7] linked the discrete formulation of Roberts to fast and efficient discrete methods for the numerical simulation of turbulent flows. We consider also this setting, since by using essentially the same techniques of the previous section we are able to prove a global existence result also for the latter model.

\subsection{Buttke loops and a discrete problem}

The main idea (refer for instance to [7, 8]) is to introduce a new variable $\overrightarrow{\mathbf{m}}$ (that is called magnetization or vortex magnetization), that is obtained by adding to the velocity $\overrightarrow{\mathbf{u}}$ a gradient at $t=0$ :

$$
\overrightarrow{\mathbf{m}}=\overrightarrow{\mathbf{u}}+\nabla q .
$$

The unknown $\overrightarrow{\mathbf{m}}$ does not satisfy the incompressibility constraint, but it is with compact support and it satisfies

$$
\operatorname{curl} \overrightarrow{\mathbf{u}}=\operatorname{curl} \overrightarrow{\mathbf{m}} .
$$

Then, we can interpret $\overrightarrow{\mathbf{m}}$ as essentially local, while $\nabla q$ is an extensive field. The decomposition (4.1) resembles the Helmholtz decomposition, even if $\overrightarrow{\mathbf{m}}$ is different from $\overrightarrow{\mathbf{u}}$, or from momentum density. The vector $\overrightarrow{\mathbf{m}}$ is related to the so-called "effective vorticity" (compactly supported) by the relation $\overrightarrow{\boldsymbol{\xi}}=\operatorname{curl} \overrightarrow{\mathbf{m}}$. Finally, the introduction of the new variable $\overrightarrow{\mathbf{m}}$ can be seen as a gauge transformation, known as "geometric gauge."

The equation of evolution for $\overrightarrow{\mathbf{m}}$ can be easily determined:

$$
\frac{\partial \overrightarrow{\mathbf{m}}}{\partial t}+(\overrightarrow{\mathbf{u}} \cdot \nabla) \overrightarrow{\mathbf{m}}+\overrightarrow{\mathbf{m}} \cdot(\nabla \overrightarrow{\mathbf{u}})^{T}=\mathbf{0}
$$

where $\overrightarrow{\mathbf{u}}=\mathbb{P} \overrightarrow{\mathbf{m}}, \mathbb{P}$ denoting the Leray projection over divergence-free vector fields. The main point is that we have an equivalent equation for $\overrightarrow{\mathbf{u}}$ plus an arbitrary gradient at the initial time. A proper choice of this gradient leads to the study of vortex loops. In fact, if curl $\overrightarrow{\mathbf{u}}$ has support within a small ball $B$, then the resulting $\overrightarrow{\mathbf{m}}$ has support within the same ball and

$$
\overrightarrow{\mathbf{m}}(\overrightarrow{\mathbf{x}})=\overrightarrow{\mathbf{M}} \rho\left(\overrightarrow{\mathbf{x}}-\overrightarrow{\mathbf{x}}_{B}\right) \quad \text { for some } \overrightarrow{\mathbf{x}}_{B} \in B .
$$

Here $\overrightarrow{\mathbf{M}}$ is a vector in $\mathbb{R}^{3}$, while $\rho$ is a non-negative smooth function with support within $B$ and such that $\int_{\mathbb{R}^{3}} \rho(\overrightarrow{\mathbf{x}}) d \overrightarrow{\mathbf{x}}=1$. The "magnet" $\overrightarrow{\mathbf{M}} \rho\left(\overrightarrow{\mathbf{x}}-\overrightarrow{\mathbf{x}}_{B}\right)$ has a simple interpretation: the velocity field induced is the same of a small vorticity loop (or vortex ring), with $\overrightarrow{\mathbf{M}}$ perpendicular to the plane of the loop and $|\overrightarrow{\mathbf{M}}|=\Gamma \pi R^{2}$. Then, from the physical point of view the vector $\overrightarrow{\mathbf{m}}$ can be interpreted as a vortex dipole density.

The above interpretation can be used to deduce a Hamiltonian system for a more complicated distribution of vorticity, see Buttke [7]. This approach works as a de-singularization 
of the problem: the resulting discrete dynamic we shall consider includes in an implicit way a regularization that will allows us to prove global existence of smooth solutions as well as good computational properties investigated in [7.

The discrete model is derived by considering a finite sum of magnets and denoting by $\overrightarrow{\mathbf{x}}_{\alpha}$, for $\alpha=1, \ldots, N$, the positions of the loops and by $\overrightarrow{\mathbf{m}}_{\alpha}$ be the corresponding magnetization vector, that is not anymore a constant vector. Then, the velocity field $\overrightarrow{\mathbf{u}}$ of the fluid is given by the solution of the equation

$$
\overrightarrow{\mathbf{u}}=\overrightarrow{\mathbf{M}}+\nabla q
$$

with

$$
\overrightarrow{\mathbf{M}}(\overrightarrow{\mathbf{x}}, t)=\sum_{\alpha=1}^{N} \overrightarrow{\mathbf{m}}_{\alpha}(t) \rho\left(\overrightarrow{\mathbf{x}}-\overrightarrow{\mathbf{x}}_{\alpha}\right),
$$

where the scalar $\phi$ is determined by the equation

$$
\Delta q=-\nabla \cdot \overrightarrow{\mathbf{M}}
$$

The equation of motion for $\overrightarrow{\mathbf{M}}$ is (component-wise)

$$
\frac{\partial \mathbf{M}^{i}}{\partial t}+\sum_{j=1}^{3} \mathbf{u}^{j} \nabla^{j} \mathbf{M}^{i}=-\sum_{j=1}^{3} \mathbf{M}^{j} \nabla^{i} \mathbf{u}^{j} \quad i=1, \ldots, 3
$$

and the "loop particles" moves according to the system of ordinary differential equations

$$
\left\{\begin{array}{l}
\dot{\mathbf{m}}_{\alpha}^{i}=-\sum_{j=1}^{3} \mathbf{m}_{\alpha}^{j} \nabla^{i} \mathbf{u}^{j}\left(\overrightarrow{\mathbf{x}}_{\alpha}\right) \\
\dot{\mathbf{x}}_{\alpha}^{i}=\mathbf{u}^{i}\left(\overrightarrow{\mathbf{x}}_{\alpha}\right),
\end{array}\right.
$$

where the index $\alpha$ run over particle labels.

Equations (4.2) form a Hamiltonian system, if we consider the conjugate pairs of variables $\left(\overrightarrow{\mathbf{x}}_{\alpha}, \overrightarrow{\mathbf{m}}_{\alpha}\right)_{\alpha=1, \ldots, N}$ with symplectic structure

$$
d \Omega=\sum_{\alpha=1}^{N} \sum_{i=1}^{3} d \mathbf{m}_{\alpha}^{i} \wedge d \mathbf{x}_{\alpha}^{i}
$$

and Hamiltonian function

$$
\mathcal{H}\left(\left\{\overrightarrow{\mathbf{x}}_{\alpha}, \overrightarrow{\mathbf{m}}_{\alpha}\right\}\right)=\frac{1}{2} \sum_{\alpha, \beta=1}^{N} \overrightarrow{\mathbf{m}}_{\alpha} \cdot \overrightarrow{\mathbf{m}}_{\beta} \rho\left(\overrightarrow{\mathbf{x}}_{\alpha}-\overrightarrow{\mathbf{x}}_{\beta}\right)+\left(\overrightarrow{\mathbf{m}}_{\alpha} \cdot \nabla\right)\left(\overrightarrow{\mathbf{m}}_{\beta} \cdot \nabla\right) \Phi\left(\overrightarrow{\mathbf{x}}_{\alpha}-\overrightarrow{\mathbf{x}}_{\beta}\right),
$$

where $\Phi$ is the solution of $\Delta \Phi=\rho$. Hence system (4.2) takes the canonical form

$$
\left\{\begin{array}{l}
\dot{\overrightarrow{\mathbf{x}}}_{\alpha}=\frac{\partial \mathcal{H}}{\partial \overrightarrow{\mathbf{m}}_{\alpha}} \\
\dot{\overrightarrow{\mathbf{m}}}_{\alpha}=-\frac{\partial \mathcal{H}}{\partial \overrightarrow{\mathbf{x}}_{\alpha}} .
\end{array}\right.
$$


Remark 5 The analogy between magneto-static and fluid mechanics is that to the electric current it corresponds the vorticity and to the magnetic induction it corresponds the velocity. Since the name "magnetization" may be misleading, we prefer to use Chorin's notation and to refer to them as Buttke loops or vorticity loops.

In order to show that the energy is constant on solutions we pass to the wave-numbers notation. Let $\widehat{\rho}(\overrightarrow{\mathbf{k}})$ be the Fourier transform of $\rho(\overrightarrow{\mathbf{x}})$. The energy $\mathcal{H}\left(\left\{\overrightarrow{\mathbf{x}}_{\alpha}, \overrightarrow{\mathbf{m}}_{\alpha}\right\}\right)$ can be written, in terms of wave-numbers, as follows

$$
\mathcal{H}\left(\left\{\overrightarrow{\mathbf{x}}_{\alpha}, \overrightarrow{\mathbf{m}}_{\alpha}\right\}\right)=\frac{1}{2(2 \pi)^{3}} \int_{\mathbb{R}^{3}} \widehat{\rho}(\overrightarrow{\mathbf{k}})\left|\Pi_{\overrightarrow{\mathbf{k}}} \sum_{\alpha=1}^{N} \overrightarrow{\mathbf{m}}_{\alpha} \mathrm{e}^{i\left\langle\overrightarrow{\mathbf{k}} \overrightarrow{\mathbf{x}}_{\alpha}\right\rangle}\right|^{2} d \overrightarrow{\mathbf{k}}
$$

where $\Pi_{\overrightarrow{\mathbf{k}}}$ is the projection in the plane orthogonal to the vector $\overrightarrow{\mathbf{k}}$. From this representation it is clear that the energy is positive definite. Moreover, the velocity field has the form

$$
\overrightarrow{\mathbf{u}}(\overrightarrow{\mathbf{x}})=\frac{1}{(2 \pi)^{3}} \int_{\mathbb{R}^{3}} \widehat{\rho}(\overrightarrow{\mathbf{k}}) \Pi_{\overrightarrow{\mathbf{k}}} \sum_{\alpha=1}^{N} \overrightarrow{\mathbf{m}}_{\alpha} \mathrm{e}^{-i\left\langle\overrightarrow{\mathbf{k}}, \overrightarrow{\mathbf{x}}-\overrightarrow{\mathbf{x}}_{\alpha}\right\rangle} d \overrightarrow{\mathbf{k}}
$$

and the tensor $\nabla \overrightarrow{\mathbf{u}}$ appearing in the equation for $\overrightarrow{\mathbf{m}}$ is

$$
[\nabla \overrightarrow{\mathbf{u}}]^{l j}(\overrightarrow{\mathbf{x}})=\frac{1}{(2 \pi)^{3}} \int_{\mathbb{R}^{3}} \widehat{\rho}(\overrightarrow{\mathbf{k}})(-i \overrightarrow{\mathbf{k}})^{l} \sum_{\alpha=1}^{N}\left(\Pi_{\overrightarrow{\mathbf{k}}} \overrightarrow{\mathbf{m}}_{\alpha}\right)^{j} \mathrm{e}^{-i\left\langle\overrightarrow{\mathbf{k}}, \overrightarrow{\mathbf{x}}-\overrightarrow{\mathbf{x}}_{\alpha}\right\rangle} d \overrightarrow{\mathbf{k}} .
$$

\subsection{The periodic problem}

In order to avoid problems related with the decay at infinity and possibly non-converging integrals, we restrict to the periodic setting. Thus, we fix a box of linear size $2 \pi$ and consider the periodic version of the above problem, i.e., we substitute Fourier series expansion to the Fourier transform.

The energy is now given by

$$
\mathcal{H}\left(\left\{\overrightarrow{\mathbf{x}}_{\alpha}, \overrightarrow{\mathbf{m}}_{\alpha}\right\}\right)=\frac{1}{2(2 \pi)^{3}} \sum_{\overrightarrow{\mathbf{k}} \in \mathbb{Z}^{3}} \widehat{\rho}(\overrightarrow{\mathbf{k}})\left|\Pi_{\overrightarrow{\mathbf{k}}} \sum_{\alpha=1}^{N} \overrightarrow{\mathbf{m}}_{\alpha} \mathrm{e}^{i\left\langle\overrightarrow{\mathbf{k}}, \overrightarrow{\mathbf{x}}_{\alpha}\right\rangle}\right|^{2},
$$

where $\overrightarrow{\mathbf{k}}$ runs over $\mathbb{Z}^{3}$ and the coordinate $\overrightarrow{\mathbf{x}}_{\alpha}$ is restricted to the box $\left.\Lambda_{L}=\right]-\pi, \pi\left[{ }^{3}\right.$. The velocity is then

$$
\left.\overrightarrow{\mathbf{u}}(\overrightarrow{\mathbf{x}})=\frac{1}{(2 \pi)^{3}} \sum_{\overrightarrow{\mathbf{k}} \in \mathbb{Z}^{3}} \hat{\rho}(\overrightarrow{\mathbf{k}}) \Pi_{\overrightarrow{\mathbf{k}}} \sum_{\alpha=1}^{N} \overrightarrow{\mathbf{m}}_{\alpha} \mathrm{e}^{-i\left\langle\overrightarrow{\mathbf{k}}, \overrightarrow{\mathbf{x}}-\overrightarrow{\mathbf{x}}_{\alpha}\right\rangle} \quad \forall \overrightarrow{\mathbf{x}} \in\right]-\pi, \pi\left[\left[^{3} .\right.\right.
$$

By using standard techniques we are able to prove the following result.

Proposition 1 The solution for the evolution problem of a finite number of loops in the periodic box $\Lambda_{L}$ exists (and is unique) for any positive time, provided that the initial condition has finite energy and the function $\widehat{\rho}(\overrightarrow{\mathbf{k}})$ is non-negative and satisfies the decay estimate

$$
\sum_{\overrightarrow{\mathbf{k}} \in \mathbb{Z}^{3}}|\overrightarrow{\mathbf{k}}|^{4} \hat{\rho}(\overrightarrow{\mathbf{k}})<+\infty
$$


Remark 6 The condition of decay for $|\overrightarrow{\mathbf{k}}| \rightarrow+\infty$ required in Proposition 1 is easily satisfied if $\rho(\overrightarrow{\mathbf{x}})$ is a smooth enough function. The condition on the non-negativity of $\hat{\rho}(\overrightarrow{\mathbf{k}})$ is not automatically satisfied for each smooth function $\rho(\overrightarrow{\mathbf{x}})$. A wide class of smooth functions with compact support and non-negative (hence real) Fourier transform can be identified as follows. Consider a smooth (say $C^{\infty}$ ) "bump" function $b(\overrightarrow{\mathbf{x}})$ over the ball $B(\mathbf{0}, \delta)$ and, in addition, suppose that $b(\overrightarrow{\mathbf{x}})$ is "even," implying that the Fourier transform has vanishing imaginary part. Then, define $\rho(\overrightarrow{\mathbf{x}}):=(b * b)(\overrightarrow{\mathbf{x}})$ and this will turn out to be smooth, non-negative, and null outside the ball $B(\mathbf{0}, 2 \delta)$ of radius twice that of the original one. Finally, by using the convolution theorem it will follow that

$$
\widehat{\rho}(\overrightarrow{\mathbf{k}})=\widehat{b * b}(\overrightarrow{\mathbf{k}})=\widehat{b}(\overrightarrow{\mathbf{k}}) \cdot \widehat{b}(\overrightarrow{\mathbf{k}}) \geq 0 .
$$

Proof of Proposition 1. Again the proof is based on an energy estimate and an a-priori bound of the velocity in terms of the energy. The main point of the proof is the following Cauchy-Schwartz inequality:

$$
\begin{aligned}
& |\overrightarrow{\mathbf{u}}(\overrightarrow{\mathbf{x}})| \leq \frac{1}{(2 \pi)^{3}} \sum_{\overrightarrow{\mathbf{k}} \in \mathbb{Z}^{3}} \widehat{\rho}(\overrightarrow{\mathbf{k}})\left|\Pi_{\overrightarrow{\mathbf{k}}} \sum_{\alpha=1}^{N} \overrightarrow{\mathbf{m}}_{\alpha} \mathrm{e}^{-i\left\langle\overrightarrow{\mathbf{k}}, \overrightarrow{\mathbf{x}}-\overrightarrow{\mathbf{x}}_{\alpha}\right\rangle}\right|
\end{aligned}
$$

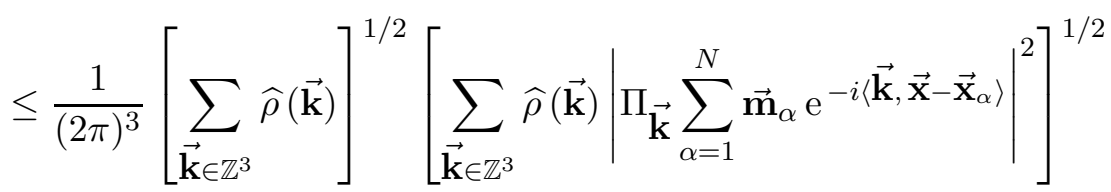

$$
\begin{aligned}
& =\frac{1}{2 \pi^{3 / 2}}\left[\sum_{\overrightarrow{\mathbf{k}} \in \mathbb{Z}^{3}} \widehat{\rho}(\overrightarrow{\mathbf{k}})\right]^{1 / 2} \mathcal{H}^{1 / 2}\left(\left\{\overrightarrow{\mathbf{x}}_{\alpha}, \overrightarrow{\mathbf{m}}_{\alpha}\right\}\right) \text {. }
\end{aligned}
$$

and in the same way it easily follows that

$$
\|\overrightarrow{\mathbf{u}}\|+\|\nabla \overrightarrow{\mathbf{u}}\|+\left\|\nabla^{2} \overrightarrow{\mathbf{u}}\right\| \leq \frac{1}{2 \pi^{3 / 2}}\left[\sum_{\overrightarrow{\mathbf{k}} \in \mathbb{Z}^{3}}|\overrightarrow{\mathbf{k}}|^{4} \widehat{\rho}(\overrightarrow{\mathbf{k}})\right]^{1 / 2} \mathcal{H}^{1 / 2}\left(\left\{\overrightarrow{\mathbf{x}}_{\alpha}, \overrightarrow{\mathbf{m}}_{\alpha}\right\}\right),
$$

showing that we can control the regularity of $\overrightarrow{\mathbf{u}}$ in terms of the energy $\mathcal{H}\left(\left\{\overrightarrow{\mathbf{x}}_{\alpha}, \overrightarrow{\mathbf{m}}_{\alpha}\right\}\right)$. By a direct differentiation it is also clear that $\mathcal{H}\left(\left\{\overrightarrow{\mathbf{x}}_{\alpha}, \overrightarrow{\mathbf{m}}_{\alpha}\right\}\right)$ is constant along (smooth enough) solutions of the equations (4.2) so that we have good control over $\overrightarrow{\mathbf{u}}$ for any time. This implies the global existence of solutions.

\section{A class of discrete models of evolution of vorticity}

In this section we study a class of discrete models for the evolution of vorticity which includes discrete versions of the line vortex model. These models could be of interest in the numerical simulation using lattice methods as suggested in $[8$. In the sequel we shall always assume on the function $\varphi$ the four natural hypotheses (A.1)-(A.4), we introduced in Section 2.2

We consider distribution of vorticity giving rise to a velocity field of the form

$$
\overrightarrow{\mathbf{u}}(\overrightarrow{\mathbf{x}})=\sum_{\alpha=1}^{N} \nabla \varphi\left(\overrightarrow{\mathbf{x}}-\overrightarrow{\mathbf{x}}_{\alpha}\right) \wedge \overrightarrow{\boldsymbol{\xi}}_{\alpha}
$$


that is parametrized by the set of $6 N$ variables $\left\{\overrightarrow{\mathbf{x}}_{\alpha}, \overrightarrow{\boldsymbol{\xi}}_{\alpha}\right\}_{\alpha=1, \ldots, N}$. The physical interpretation of this equation is that of describing the superposition of $N$ vortex "blobs" (i.e. concentrations of vorticity) indexed by $\alpha$, situated at points $\overrightarrow{\mathbf{x}}_{\alpha}$, and whose vorticity is directed along the vector $\overrightarrow{\boldsymbol{\xi}}_{\alpha}$. In this case these blobs determine the initial datum and there are no assumptions that the solution persists being of the same type for all positive times. This is the difference between blobs and loops considered in the previous section.

A priori we can think to each of the variables $\left\{\overrightarrow{\mathbf{x}}_{\alpha}, \overrightarrow{\boldsymbol{\xi}}_{\alpha}\right\}_{\alpha}$ as an independent degree of freedom. However, if we set $\overrightarrow{\boldsymbol{\xi}}_{\alpha}=\overrightarrow{\mathbf{x}}_{\alpha+1}-\overrightarrow{\mathbf{x}}_{\alpha}$ (with the understanding that $\alpha+1=0$ if $\alpha=N)$, then this model corresponds to a natural discretization of the line vortex model previously described.

For the moment we do not impose any constraint on the variables $\overrightarrow{\boldsymbol{\xi}}$ (either static or dynamic) and we just impose that the points $\overrightarrow{\mathbf{x}}_{\alpha}$ are transported by the flow $\overrightarrow{\mathbf{u}}$ so that we have

$$
\dot{\overrightarrow{\mathbf{x}}}_{\alpha}=\overrightarrow{\mathbf{u}}\left(\overrightarrow{\mathbf{x}}_{\alpha}\right), \quad \alpha=1, \ldots, N .
$$

This is the discrete counterpart of (1.3). If we want to address the problem of the global (in time) dynamics of these models we can introduce the following useful quantities

$$
\mathcal{L}=\sum_{\alpha=1}^{N}\left|\overrightarrow{\boldsymbol{\xi}}_{\alpha}\right| \quad \text { and } \quad \mathcal{A}=\sum_{\alpha=1}^{N}\left|\overrightarrow{\boldsymbol{\xi}}_{\alpha}\right|^{2}
$$

that are respectively the length and the quadratic variation of the curve itself. Moreover, in analogy with the Hamiltonian introduced in Section 3, we define an energy function (which controls the magnitude and regularity of the velocity field $\overrightarrow{\mathbf{u}}$ ) as follows:

$$
\mathcal{H}\left(\left\{\overrightarrow{\mathbf{x}}_{\alpha}, \overrightarrow{\boldsymbol{\xi}}_{\alpha}\right\}\right)=\frac{1}{2} \sum_{\alpha, \beta=1}^{N} \varphi\left(\overrightarrow{\mathbf{x}}_{\alpha}-\overrightarrow{\mathbf{x}}_{\beta}\right)\left\langle\overrightarrow{\boldsymbol{\xi}}_{\alpha}, \overrightarrow{\boldsymbol{\xi}}_{\beta}\right\rangle
$$

It is not difficult to show that we have an analogous of Lemma 3, which allows us to control $\overrightarrow{\mathbf{u}}$ in term of $\mathcal{H}$ and, of course, we have the following trivial bounds

$$
\left\|\nabla^{n} \overrightarrow{\mathbf{u}}\right\|_{L^{\infty}} \leq c_{n, \varphi} \mathcal{L}, \quad \text { and } \quad \mathcal{H} \leq\|\varphi\|_{L^{\infty}} \mathcal{L}^{2}
$$

where the constants $c_{n, \varphi}$ depends only on $\varphi$ and the number $n$ of derivative (we assume that $\varphi$ is sufficiently regular for all the constants in this section to be well defined. For example it is enough to take $\varphi$ infinitely differentiable.)

Let us now compute the time derivative of the energy. Taking into account eq. (5.1), but leaving the chance of an arbitrary time-derivative $\partial_{t} \overrightarrow{\boldsymbol{\xi}}_{\alpha}$ for $\overrightarrow{\boldsymbol{\xi}}_{\alpha}$, we obtain

$$
\frac{d \mathcal{H}\left(\left\{\overrightarrow{\mathbf{x}}_{\alpha}, \overrightarrow{\boldsymbol{\xi}}_{\alpha}\right\}\right)}{d t}=\sum_{\alpha, \beta=1}^{N} \varphi\left(\overrightarrow{\mathbf{x}}_{\alpha}-\overrightarrow{\mathbf{x}}_{\beta}\right)\left\langle\overrightarrow{\boldsymbol{\xi}}_{\alpha}, \partial_{t} \overrightarrow{\boldsymbol{\xi}}_{\beta}\right\rangle-\sum_{\alpha, \beta=1}^{N} \overrightarrow{\mathbf{u}}\left(\overrightarrow{\mathbf{x}}_{\beta}\right) \cdot \nabla \varphi\left(\overrightarrow{\mathbf{x}}_{\alpha}-\overrightarrow{\mathbf{x}}_{\beta}\right)\left\langle\overrightarrow{\boldsymbol{\xi}}_{\alpha}, \overrightarrow{\boldsymbol{\xi}}_{\beta}\right\rangle .
$$

We now analyze the second sum appearing in the above equality, in order to recover some 
symmetries or cancellation properties. We get

$$
\begin{aligned}
I & =\sum_{\alpha, \beta=1}^{N} \overrightarrow{\mathbf{u}}\left(\overrightarrow{\mathbf{x}}_{\beta}\right) \cdot \nabla \varphi\left(\overrightarrow{\mathbf{x}}_{\alpha}-\overrightarrow{\mathbf{x}}_{\beta}\right)\left\langle\overrightarrow{\boldsymbol{\xi}}_{\alpha}, \overrightarrow{\boldsymbol{\xi}}_{\beta}\right\rangle-\sum_{\alpha, \beta=1}^{N} \overrightarrow{\boldsymbol{\xi}}_{\alpha} \cdot \overrightarrow{\mathbf{u}}\left(\overrightarrow{\mathbf{x}}_{\beta}\right) \overrightarrow{\boldsymbol{\xi}}_{\beta} \cdot \nabla \varphi\left(\overrightarrow{\mathbf{x}}_{\alpha}-\overrightarrow{\mathbf{x}}_{\beta}\right) \\
& =\sum_{\alpha, \beta=1}^{N}\left\langle\overrightarrow{\boldsymbol{\xi}}_{\alpha} \wedge \nabla \varphi\left(\overrightarrow{\mathbf{x}}_{\alpha}-\overrightarrow{\mathbf{x}}_{\beta}\right), \overrightarrow{\boldsymbol{\xi}}_{\beta} \wedge \overrightarrow{\mathbf{u}}\left(\overrightarrow{\mathbf{x}}_{\beta}\right)\right\rangle \\
& =-\sum_{\beta=1}^{N}\left\langle\overrightarrow{\mathbf{u}}\left(\overrightarrow{\mathbf{x}}_{\beta}\right),\left[\overrightarrow{\boldsymbol{\xi}}_{\beta} \wedge \overrightarrow{\mathbf{u}}\left(\overrightarrow{\mathbf{x}}_{\beta}\right)\right]\right\rangle=0
\end{aligned}
$$

and this implies that we can write the following equivalent expression for the time derivative of $\mathcal{H}\left(\left\{\overrightarrow{\mathbf{x}}_{\alpha}, \overrightarrow{\boldsymbol{\xi}}_{\alpha}\right\}\right)$ :

$$
\frac{d \mathcal{H}\left(\left\{\overrightarrow{\mathbf{x}}_{\alpha}, \overrightarrow{\boldsymbol{\xi}}_{\alpha}\right\}\right)}{d t}=\sum_{\alpha, \beta=1}^{N} \varphi\left(\overrightarrow{\mathbf{x}}_{\alpha}-\overrightarrow{\mathbf{x}}_{\beta}\right)\left\langle\overrightarrow{\boldsymbol{\xi}}_{\alpha}, \partial_{t} \overrightarrow{\boldsymbol{\xi}}_{\beta}\right\rangle-\sum_{\alpha, \beta=1}^{N}\left\langle\overrightarrow{\boldsymbol{\xi}}_{\alpha}, \overrightarrow{\mathbf{u}}\left(\overrightarrow{\mathbf{x}}_{\beta}\right)\right\rangle\left\langle\overrightarrow{\boldsymbol{\xi}}_{\beta}, \nabla \varphi\left(\overrightarrow{\mathbf{x}}_{\alpha}-\overrightarrow{\mathbf{x}}_{\beta}\right)\right\rangle
$$

Consider the second term and write

$$
\begin{aligned}
A & =\sum_{\alpha, \beta=1}^{N}\left\langle\overrightarrow{\boldsymbol{\xi}}_{\alpha}, \overrightarrow{\mathbf{u}}\left(\overrightarrow{\mathbf{x}}_{\beta}\right)\right\rangle\left\langle\overrightarrow{\boldsymbol{\xi}}_{\beta}, \nabla \varphi\left(\overrightarrow{\mathbf{x}}_{\alpha}-\overrightarrow{\mathbf{x}}_{\beta}\right)\right\rangle \\
& =-\sum_{\beta=1}^{N}\left\langle\overrightarrow{\boldsymbol{\xi}}_{\beta}, \nabla \Phi\left(\overrightarrow{\mathbf{x}}_{\beta}\right)\right\rangle+\sum_{\alpha, \beta=1}^{N} \sum_{i, j=1}^{3} \varphi\left(\overrightarrow{\mathbf{x}}_{\alpha}-\overrightarrow{\mathbf{x}}_{\beta}\right) \boldsymbol{\xi}_{\alpha}^{i} \nabla^{j} \mathbf{u}^{i}\left(\overrightarrow{\mathbf{x}}_{\beta}\right) \boldsymbol{\xi}_{\beta}^{j}
\end{aligned}
$$

where

$$
\Phi(\overrightarrow{\mathbf{x}})=\sum_{\alpha=1}^{N} \varphi\left(\overrightarrow{\mathbf{x}}-\overrightarrow{\mathbf{x}}_{\alpha}\right)\left\langle\overrightarrow{\boldsymbol{\xi}}_{\alpha}, \overrightarrow{\mathbf{u}}(\overrightarrow{\mathbf{x}})\right\rangle .
$$

Finally, the rate of change of the energy takes the form

$$
\frac{d}{d t} \mathcal{H}\left(\left\{\overrightarrow{\mathbf{x}}_{\alpha}, \overrightarrow{\boldsymbol{\xi}}_{\alpha}\right\}\right)=\sum_{\alpha, \beta=1}^{N} \sum_{i, j=1}^{3} \varphi\left(\overrightarrow{\mathbf{x}}_{\alpha}-\overrightarrow{\mathbf{x}}_{\beta}\right) \boldsymbol{\xi}_{\alpha}^{i}\left[\partial_{t} \boldsymbol{\xi}_{\beta}^{i}-\nabla^{j} \mathbf{u}^{i}\left(\overrightarrow{\mathbf{x}}_{\beta}\right) \boldsymbol{\xi}_{\beta}^{j}\right]+\sum_{\beta=1}^{N} \overrightarrow{\boldsymbol{\xi}}_{\beta} \cdot \nabla \Phi\left(\overrightarrow{\mathbf{x}}_{\beta}\right) .
$$

We can rewrite the above expression by setting $\overrightarrow{\boldsymbol{\Psi}}(\overrightarrow{\mathbf{x}})=\sum_{\alpha=1}^{N} \varphi\left(\overrightarrow{\mathbf{x}}-\overrightarrow{\mathbf{x}}_{\alpha}\right) \overrightarrow{\boldsymbol{\xi}}_{\alpha}$ hence obtaining

$$
\frac{d}{d t} \mathcal{H}\left(\left\{\overrightarrow{\mathbf{x}}_{\alpha}, \overrightarrow{\boldsymbol{\xi}}_{\alpha}\right\}\right)=\sum_{\beta=1}^{3} \sum_{i, j=1}^{3} \boldsymbol{\Psi}^{i}\left(\overrightarrow{\mathbf{x}}_{\beta}\right)\left[\partial_{t} \boldsymbol{\xi}_{\beta}^{i}-\nabla^{j} \mathbf{u}^{i}\left(\overrightarrow{\mathbf{x}}_{\beta}\right) \boldsymbol{\xi}_{\beta}^{j}\right]+\sum_{\beta=1}^{N} \overrightarrow{\boldsymbol{\xi}}_{\beta} \cdot \nabla \Phi\left(\overrightarrow{\mathbf{x}}_{\beta}\right),
$$

with $\Phi=\overrightarrow{\boldsymbol{\Psi}} \cdot \operatorname{curl} \overrightarrow{\boldsymbol{\Psi}}$ since, by definition, $\overrightarrow{\mathbf{u}}(\overrightarrow{\mathbf{x}})=\operatorname{curl} \overrightarrow{\boldsymbol{\Psi}}(\overrightarrow{\mathbf{x}})$. By using a first order Taylor expansion with integral remainder, the introduction of the auxiliary function $\Phi$ allows us to rewrite the second term of (5.2) as follows:

$$
\sum_{\beta=1}^{N} \overrightarrow{\boldsymbol{\xi}}_{\beta} \cdot \nabla \Phi\left(\overrightarrow{\mathbf{x}}_{\beta}\right)=\sum_{\beta=1}^{N}\left[\Phi\left(\overrightarrow{\mathbf{x}}_{\beta}+\overrightarrow{\boldsymbol{\xi}}_{\beta}\right)-\Phi\left(\overrightarrow{\mathbf{x}}_{\beta}\right)\right]+\sum_{\beta=1}^{N}\left\langle\overrightarrow{\boldsymbol{\xi}}_{\beta}, \Theta_{\beta} \overrightarrow{\boldsymbol{\xi}}_{\beta}\right\rangle
$$


where $\Theta_{\beta}$ is the matrix

$$
\Theta_{\beta}^{i j}=\int_{0}^{1}(1-\rho) \nabla^{j} \nabla^{i} \Phi\left(\overrightarrow{\mathbf{x}}_{\beta}+\rho \overrightarrow{\boldsymbol{\xi}}_{\beta}\right) d \rho
$$

We observe that analogously to Lemma 3 we can control the function $\overrightarrow{\boldsymbol{\Psi}}$ in terms of the energy $\mathcal{H}$ according to the following lemma, whose straightforward proof is left to the reader.

Lemma 4 For any $0 \leq n \in \mathbb{N}$, we have the bound

$$
\left\|\nabla^{n} \overrightarrow{\boldsymbol{\Psi}}\right\|_{L^{\infty}} \leq\left[\int_{\mathbb{R}^{3}}|\overrightarrow{\mathbf{k}}|^{2 n} \widehat{\varphi}(\overrightarrow{\mathbf{k}}) d \overrightarrow{\mathbf{k}}\right]^{1 / 2} \mathcal{H}^{1 / 2}\left(\left\{\overrightarrow{\mathbf{x}}_{\alpha}, \overrightarrow{\boldsymbol{\xi}}_{\alpha}\right\}\right) .
$$

In the same way we can bound $\Phi$ since, e.g., it holds

$$
\|\Phi\|_{L^{\infty}}=\|\vec{\Psi} \cdot \operatorname{curl} \overrightarrow{\boldsymbol{\Psi}}\|_{L^{\infty}} \leq c_{\varphi} \mathcal{H}
$$

where $c_{\varphi}$ is some positive constant depending just on the Sobolev regularity of $\varphi$.

At this point (see also 8] ) we may recognize to have two interesting cases:

A) The discrete filament model, obtained by setting $\overrightarrow{\boldsymbol{\xi}}_{\alpha}=\overrightarrow{\mathbf{x}}_{\alpha+1}-\overrightarrow{\mathbf{x}}_{\alpha}$. In this case the first term in the r.h.s. of Eq. (5.3) vanishes due to the fact that

$$
\sum_{\beta=1}^{N}\left[\Phi\left(\overrightarrow{\mathbf{x}}_{\beta}+\overrightarrow{\boldsymbol{\xi}}_{\beta}\right)-\Phi\left(\overrightarrow{\mathbf{x}}_{\beta}\right)\right]=\sum_{\beta=1}^{N}\left[\Phi\left(\overrightarrow{\mathbf{x}}_{\beta+1}\right)-\Phi\left(\overrightarrow{\mathbf{x}}_{\beta}\right)\right]=0 .
$$

B) A blob model, obtained by considering each couple $\left(\overrightarrow{\mathbf{x}}_{\alpha}, \overrightarrow{\boldsymbol{\xi}}_{\alpha}\right)$ as describing (an approximation of) a vortex blob and for which the dynamics of the $\overrightarrow{\boldsymbol{\xi}}_{\alpha}$ is fixed by prescribing that these vectors are transported by the flow at the point $\overrightarrow{\mathbf{x}}_{\alpha}$, i.e.

$$
\dot{\overrightarrow{\boldsymbol{\xi}}}_{\alpha}=\left(\nabla \overrightarrow{\mathbf{u}}\left(\overrightarrow{\mathbf{x}}_{\alpha}\right)\right)^{T} \cdot \overrightarrow{\boldsymbol{\xi}}_{\alpha}
$$

In this case the first term in the r.h.s. of Eq. (5.2) vanishes identically.

For these two models we prove the following result:

Lemma 5 In the discrete filament model we have

$$
\left|\partial_{t} \mathcal{H}\right| \leq c_{1} \mathcal{H} \mathcal{A}+c_{2} \mathcal{H}^{1 / 2} \mathcal{A} .
$$

In the blob model we have

$$
\left|\partial_{t} \mathcal{H}\right| \leq c_{3} N \mathcal{H}^{1 / 2}+c_{4} \mathcal{H}^{1 / 2} \mathcal{A} \quad\left|\partial_{t} \mathcal{H}\right| \leq c_{5} \mathcal{L H}^{1 / 2} .
$$

Moreover, in both models we have

$$
\left|\partial_{t} \mathcal{A}\right| \leq c_{6} \mathcal{H}^{1 / 2} \mathcal{A} \quad\left|\partial_{t} \mathcal{L}\right| \leq c_{7} \mathcal{H}^{1 / 2} \mathcal{L} .
$$

The positive constants $c_{i}$, for $i=1, \ldots, 7$, depend only on the function $\varphi$. 
Proof. Given Lemma 4 the only nontrivial part is to justify the first term appearing in the bound for the discrete filament model, i.e., $c_{1} \mathcal{H} \mathcal{A}$. This term comes form the expression

$$
G=\sum_{\beta=1}^{N} \sum_{i, j=1}^{3} \boldsymbol{\Psi}^{i}\left(\overrightarrow{\mathbf{x}}_{\beta}\right)\left[\partial_{t} \boldsymbol{\xi}_{\beta}^{i}-\nabla^{j} \mathbf{u}^{i}\left(\overrightarrow{\mathbf{x}}_{\beta}\right) \boldsymbol{\xi}_{\beta}^{j}\right]
$$

appearing as the first term in the r.h.s. of eq. (5.2). In the discrete filament model we have (note that $\overrightarrow{\boldsymbol{\xi}}$ depends just on $t$, hence the partial derivative is in fact a total derivative)

$$
\dot{\overrightarrow{\boldsymbol{\xi}}}_{\alpha}=\dot{\overrightarrow{\mathbf{x}}}_{\alpha+1}-\dot{\overrightarrow{\mathbf{x}}}_{\alpha}=\overrightarrow{\mathbf{u}}\left(\overrightarrow{\mathbf{x}}_{\alpha+1}\right)-\overrightarrow{\mathbf{u}}\left(\overrightarrow{\mathbf{x}}_{\alpha}\right)
$$

and, by a first order Taylor expansion, we get

$$
\dot{\overrightarrow{\boldsymbol{\xi}}}_{\alpha}=\left[\nabla \overrightarrow{\mathbf{u}}\left(\overrightarrow{\mathbf{x}}_{\alpha}\right)\right]^{T} \cdot \overrightarrow{\boldsymbol{\xi}}_{\alpha}+\widetilde{\Theta}_{\alpha}\left(\overrightarrow{\boldsymbol{\xi}}_{\alpha} \otimes \overrightarrow{\boldsymbol{\xi}}_{\alpha}\right),
$$

where the tensor $\widetilde{\Theta}_{\alpha}$ is given by the formula

$$
\widetilde{\Theta}_{\alpha}^{k i j}=\int_{0}^{1}(1-\rho) \nabla^{i} \nabla^{j} \overrightarrow{\mathbf{u}}^{k}\left(\overrightarrow{\mathbf{x}}_{\alpha}+\rho \overrightarrow{\boldsymbol{\xi}}_{\alpha}\right) d \rho .
$$

Then, we can bound $G$ as follows:

$$
\begin{aligned}
|G| & \leq\|\overrightarrow{\boldsymbol{\Psi}}\|_{L^{\infty}} \sum_{\beta=1}^{N} \sum_{i, j=1}^{3}\left|\partial_{t} \boldsymbol{\xi}_{\beta}^{i}-\nabla^{j} \mathbf{u}^{i}\left(\overrightarrow{\mathbf{x}}_{\beta}\right) \boldsymbol{\xi}_{\beta}^{j}\right|=\|\overrightarrow{\boldsymbol{\Psi}}\|_{L^{\infty}} \sum_{\beta=1}^{N}\left|\widetilde{\Theta}_{\beta}\left(\overrightarrow{\boldsymbol{\xi}}_{\beta} \otimes \overrightarrow{\boldsymbol{\xi}}_{\beta}\right)\right| \\
& \leq\|\overrightarrow{\boldsymbol{\Psi}}\|_{L^{\infty}} \sum_{\beta=1}^{N}\left|\overrightarrow{\boldsymbol{\xi}}_{\beta}\right|^{2} \sup _{i j k}\left|\widetilde{\Theta}_{\beta}^{i j k}\right| \leq\|\overrightarrow{\boldsymbol{\Psi}}\|_{L^{\infty}}\|\nabla \overrightarrow{\mathbf{u}}\|_{L^{\infty}} \sum_{\beta=1}^{N}\left|\overrightarrow{\boldsymbol{\xi}}_{\beta}\right|^{2} \\
& \leq c_{1} \mathcal{H} \mathcal{A} .
\end{aligned}
$$

The other bounds are proved similarly.

Remark 7 We note that the term $c_{3} N \mathcal{H}^{1 / 2}$ in the rate of energy change of the blobs model derives from the trivial bound

$$
\left|\sum_{\beta=1}^{N}\left[\Phi\left(\overrightarrow{\mathbf{x}}_{\beta}+\overrightarrow{\boldsymbol{\xi}}_{\beta}\right)-\Phi\left(\overrightarrow{\mathbf{x}}_{\beta}\right)\right]\right| \leq 2 N\|\Phi\|_{L^{\infty}}
$$

which, however, misses any possible cancellation property of the various terms. Heuristically we expect that, if the evolution is constrained in a bounded volume (e.g. by imposing periodic boundary conditions on a torus), the distribution of the set of points $\left\{\overrightarrow{\mathbf{x}}_{\alpha}\right\}_{\alpha}$ should not differ significantly from that of the points $\left\{\overrightarrow{\mathbf{x}}_{\alpha}+\overrightarrow{\boldsymbol{\xi}}_{\alpha}\right\}_{\alpha}$ and thus that the two sums $\sum_{\alpha} \Phi\left(\overrightarrow{\mathbf{x}}_{\alpha}\right)$ and $\sum_{\alpha} \Phi\left(\overrightarrow{\mathbf{x}}_{\alpha}+\overrightarrow{\boldsymbol{\xi}}_{\alpha}\right)$ should not be too different (at least not of the order of $N$ ). 


\subsection{Heuristics about adaptive numerical algorithms for vortex filaments}

The results of the previous section suggest a strategy to implement a numerical algorithm which approximates the global solution of the filament equation. Here we do not pretend mathematical rigor but only lay down some possible developments of the main results of this paper.

So let us imagine to start a numerical approximation of the vortex model with some arbitrary discretization level (with $N$ points) and follow the solution up to the instant when the variable $\mathcal{A}(t)$ is larger than a fixed value, e.g. 1. At this point proceed to a refinement of the discretization up to level $M>N$ in order to ensure that $\mathcal{A}(t)$ become lower than $1 / 2$ and continue the simulation. Upon increased discretization the energy change, but it is not difficult to argue that this change will be small (since in the limit of infinite discretization the energy will converge to a limit). So in first approximation we will neglect this change (actually is possible to devise a discrete model where the filament is replaced by a piece-wise approximation, in which, upon refinement, the energy does not change at all). In this way we ensure that, at any time $\mathcal{A}(t) \leq 1$ and so we have also an (approximate) bound for the growth of $\mathcal{H}$ :

$$
\mathcal{H}(t) \leq \mathcal{H}(0) \mathrm{e}^{\max \left\{c_{1}, c_{2}\right\} t}
$$

which implies that there will be no blow-up of the solution. Of course since $\left|\partial_{t} \mathcal{L}\right| \leq c \mathcal{H}^{1 / 2} \mathcal{L}$ there cannot be blow-up for the length of the discrete filament as long as the energy remains finite.

This algorithm is very similar to the heuristic techniques that are already adopted in numerical simulations of vortex filament dynamics, but here we can identify a clear quantitative criterion when to perform the refinement.

Recall also that the rate of change of energy is, in this case, given by

$$
\frac{d}{d t} \mathcal{H}\left(\left\{\overrightarrow{\mathbf{x}}_{\alpha}, \overrightarrow{\boldsymbol{\xi}}_{\alpha}\right\}\right)=\sum_{\beta=0}^{N}\left\langle\overrightarrow{\boldsymbol{\Psi}}\left(\overrightarrow{\mathbf{x}}_{\beta}\right), \widetilde{\Theta}_{\beta} \overrightarrow{\boldsymbol{\xi}}_{\beta} \otimes \overrightarrow{\boldsymbol{\xi}}_{\beta}\right\rangle+\sum_{\beta=0}^{N}\left\langle\overrightarrow{\boldsymbol{\xi}}_{\beta}, \Theta_{\beta} \overrightarrow{\boldsymbol{\xi}}_{\beta}\right\rangle
$$

cfr. eq. (5.2) and Lemma 5. Then, a more refined algorithm to decide where to increase the resolution of the discretization is to find points along the filament where the quantity

$$
J_{\beta}=\left\langle\overrightarrow{\boldsymbol{\Psi}}\left(\overrightarrow{\mathbf{x}}_{\beta}\right), \widetilde{\Theta}_{\beta} \overrightarrow{\boldsymbol{\xi}}_{\beta} \otimes \overrightarrow{\boldsymbol{\xi}}_{\beta}\right\rangle+\left\langle\overrightarrow{\boldsymbol{\xi}}_{\beta}, \Theta_{\beta} \overrightarrow{\boldsymbol{\xi}}_{\beta}\right\rangle
$$

is large. These points are responsible for large positive contributions to the change of energy and thus can be associated to points of instability of the finite-element approximation of the dynamics. By refining the mesh only in the neighborhood of these points we diminish the rate of change of the energy and control the discrete evolution. Currently we do not know if the latter refinement technique is too expensive from the computational point of view.

Finally, we observe that the presence of $N$ in the first term on the r.h.s of (5.4) prevents to obtain the same result for the vortex blob model, since in this case refinement will enlarge this term.

\section{Conclusions}

We considered several classes of problems related to the evolution of special patterns of singular vorticity. Various kind of regularization have been analyzed and they are based 
either 1) on a smoother version of the Biot-Savart law, linking velocity and vorticity or either 2) a "point vortex" special expression of the solution. Under reasonable assumptions we have been able to prove global-in-time existence of solutions for these various models, employing the Hamiltonian structure of the underlying 3D Euler equations.

In the case of the vortex filament we have also been able to consider curves that are not very smooth, solving an approximation of the line vortex equation containing also the classical Rosenhead approximation for this problem. In the case of small vortex loops we solved a periodic problem involving a finite number of them.

In the final section we used these results to obtain, as by-product, some results on a couple of discrete models. The discrete models we have been able to analyze are a discrete filament model and a model of $3 \mathrm{~d}$ vorticity blobs. For the latter we have been unable to find global existence results, while for the former we linked global regularity of the limit problem (smooth curve) with a numerical procedure to properly simulate this vorticity pattern. In particular, a quantitative refinement technique has been suggested, in order to produce an effective numerical simulation for arbitrarily large times.

\section{References}

[1] R.A. Adams, Sobolev spaces, Academic Press, New York-London, 1975. Pure and Applied Mathematics, Vol. 65.

[2] J.T. Beale, T. Kato, and A. Majda, Remarks on the breakdown of smooth solutions for the 3-D Euler equations, Comm. Math. Phys. 94 (1984), no. 1, 61-66.

[3] J. Bell and D. Markus, Vorticity intensification and the transition to turbulence in the three-dimensional Euler equation, Comm. Math. Phys. 147 (1992), no. 2, 371-394.

[4] L.C. Berselli and H. Bessaih, Some results for the line vortex equation, Nonlinearity 15 (2002), no. 6, 1729-1746.

[5] H. Bessaih and F. Flandoli, A mean field result with application to 3D vortex filaments, Probabilistic methods in fluids, 22-34, World Sci. Publishing, River Edge, NJ, 2003.

[6] H. Bessaih, M. Gubinelli and F. Russo, The evolution of a random vortex filament, Ann. Prob. 33 (2005), no. 5, 1825-1855.

[7] T.F. Buttke, Velicity methods: Lagrangian numerical methods which preserve the Hamiltonian structure of incompressible fluid flow. Vortex flows and related numerical methods (Grenoble, 1992), 39-57, NATO Adv. Sci. Inst. Ser. C Math. Phys. Sci., 395, Kluwer Acad. Publ., Dordrecht, 1993.

[8] A.J. Chorin, Vorticity and turbulence, Springer-Verlag, New York, 1994.

[9] P. Constantin, Geometric statistics in turbulence, SIAM Rev. 36 (1994), no. 1, 73-98.

[10] P. Constantin, Near identity transformations for the Navier-Stokes equations. Handbook of mathematical fluid dynamics, Vol. II, 117-141, North-Holland, Amsterdam, 2003

[11] P. Constantin, A.J. Majda, and E. Tabak, Formation of strong fronts in the 2-D quasigeostrophic thermal active scalar, Nonlinearity 7 (1994), no. 6, 1495-1533. 
[12] A. Córdoba and D. Córdoba, A maximum principle applied to quasi-geostrophic equations, Comm. Math. Phys. 249 (2004), no. 3, 511-528.

[13] A. Córdoba, D. Córdoba, C.L. Fefferman, and M.A. Fontelos, A geometrical constraint for capillary jet breakup, Adv. Math. 187 (2004), no. 1, 228-239.

[14] G.-H. Cottet and P.D. Koumoutsakos, Vortex methods, theory and practice, Cambridge Univ. Press, Cambridge, 2002.

[15] F. Flandoli, A probabilistic description of small scale structures in $3 D$ fluids, Ann. Inst. H. Poincaré Probab. Statist. 38 (2002), no 2, 207-228.

[16] F. Flandoli, M. Gubinelli, Gibbs ensembles of vortex filaments, Probab. Theory Related Fields, 22 (2002), no. 3, 317-340.

[17] U. Frisch, Turbulence. The legacy of A.N. Kolmogorov, Cambridge Univ. Press, Cambridge, 1995.

[18] G. Gallavotti, Foundations of fluid dynamics. Translated from the Italian. Texts and Monographs in Physics. Springer-Verlag, Berlin, 2002. xviii+513

[19] H. Hasimoto, A soliton on a vortex filament, J. Fluid. Mech. 51, (1972) 477-485.

[20] H. Helmholtz, Uber integrale der hydrodynamischen gleichungen welche den Wirbelbewegungen entsprechen, Crelle J. 55 (1885), 25.

[21] D.D. Holm, Rasetti-Regge Dirac bracket formulation of Lagrangian fluid dynamics of vortex filaments. Nonlinear waves: computation and theory, II (Athens, GA, 2001). Math. Comput. Simulation 62 (2003), no. 1-2, 53-63.

[22] Lord Kelvin (Sir William Thomson), On vortex motion, Trans. Royal Soc. Edin. 25 (1869), 217-260.

[23] R. Klein and A.J. Majda, Self-stretching of a perturbed vortex filament. I. The asymptotic equation for deviations from a straight line, Phys. D 49 (1991), no. 3, 323-352.

[24] R. Klein and A.J. Majda, Self-stretching of a perturbed vortex filament. II. Structure of solutions, Phys. D 53 (1991), no. 2-4, 267-294.

[25] P.L. Lions, On Euler equations and statistical physics, Scuola Normale Superiore, Pisa, 1997.

[26] P.L. Lions and A.J. Majda, Equilibrium statistical theory for nearly parallel vortex filaments, Comm. Pure Appl. Math, 53 (2000), no. 1, 76-142.

[27] T.J. Lyons, Differential equations driven by rough signals, Rev. Mat. Iberoamericana 14 (1998), no. 2, 215-310.

[28] T.J. Lyons and Z. Qian, System control and rough paths. Oxford Mathematical Monographs. Oxford Univ. Press, Oxford, 2002. x+216 pp.

[29] J.E. Marsden and A. Weinstein, Coadjoint orbits, vortices, and Clebsch variables for incompressible fluids. Order in chaos (Los Alamos, N.M., 1982). Phys. D 7 (1983), no. $1-3,305-323$. 
[30] D.W. Moore, Finite amplitude waves on aircraft trailing vortices, Aero. Quarterly 23 (1972), 307-314.

[31] P. Roberts, A Hamiltonian theory for weakly interacting vortices, Mathematika 19 (1972), 169-179.

[32] L. Rosenhead, The spread of vorticity in the wake behind a cylinder, Proc. Royal Soc. 127 (1930), 590-612.

[33] P.G. Saffman, Vortex dynamics, Cambridge Univ. Press, Cambridge, 1992.

[34] V.I. Osedelets, On a new way of writing the Navier-Stokes equation: the Hamiltonian formalism, Russ. Math. Surv. 44 (1988), 210-211.

[35] A. Vincent and M. Meneguzzi, The spatial structure and the statistical properties of homogeneous turbulence, J. Fluid. Mech. 225 (1991), no. 1, 1-25.

[36] W. Wolibner, Un théoreme sur l'existence du mouvement plan d'un fluide parfait homogène incompressible, pendant un temps infiniment longue, Math. Z. 37 (1933), 698726.

[37] L.C. Young, An inequality of Hölder type, connected with Stieltjes integration, Acta Math. 67 (1936) 251-282.

[38] V.I. Yudovich, Non stationary flow of an ideal incompressible liquid, Comput. Math. \& Math. Phys. 3 (1963), 1407-1456. (Russian). 